\title{
Kernos
}

Revue internationale et pluridisciplinaire de religion grecque antique

19 | 2006

Varia

\section{Hésiode, Homère, Hérodote : forme catalogique et classifications génériques}

Ioanna Papadopoulou-Belmehdi

\section{(2) OpenEdition}

\section{Journals}

Édition électronique

URL : https://journals.openedition.org/kernos/432

DOI : $10.4000 /$ kernos.432

ISSN : 2034-7871

\section{Éditeur}

Centre international d'étude de la religion grecque antique

\section{Édition imprimée}

Date de publication : 1 janvier 2006

Pagination : 79-95

ISSN : 0776-3824

\section{Référence électronique}

Ioanna Papadopoulou-Belmehdi, «Hésiode, Homère, Hérodote : forme catalogique et classifications génériques », Kernos [En ligne], 19 | 2006, mis en ligne le 20 mars 2011, consulté le 24 août 2022. URL http://journals.openedition.org/kernos/432 ; DOI : https://doi.org/10.4000/kernos.432 


\title{
Hésiode, Homère, Hérodote : forme catalogique et classifications génériques
}

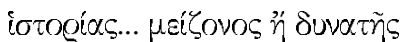

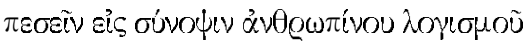 \\ Denys d'Halicarnasse, VII, 5, 5
}

\begin{abstract}
Résumé : L’usage de la forme catalogique et de la généalogie (envisagée ici comme souscatégorie de la forme catalogique) inscrit les auteurs dans une filiation intellectuelle. Partant de cette hypothèse, l'article réexamine la question de l'influence homérique sur l'œuvre d'Hérodote (surtout à partir des livres II et VII) face à l'influence hésiodique sur la naissance de l'historiographie. L'objectif est de montrer que la manipulation de la forme catalogique est un critère d'innovation autant de l'école homérique face à l'école hésiodique que d'Hérodote face au style historiographique dominant. Les options poétiques «originelles» divergentes semblent ainsi avoir exercé une influence durable sur le processus de la formation des genres en Grèce ancienne.

Abstract: Departing from the hypothesis that the use of catalogues and genealogies (considered here as a sub-category of the catalogic form) assigns authors to an intellectual genealogy, this paper re-examines the Homeric influence on Herodotus (especially in books II and VII) compared to the Hesiodic influence on the beginnings of historiography. The use of catalogues can be envisaged as a criterion of innovation, when one compares on this point the Homeric and Hesiodic "schools" as well as Herodotus and contemporary historiographers, and shows the lasting influence of the originally divergent poetic options on the process of the formation of genres in ancient Greece.
\end{abstract}

Le statut de la forme catalogique dans l'histoire des genres discursifs grecs constitue un problème ouvert, relativement négligé, mais qui ne relève pas moins des questions fondamentales de nos disciplines. À commencer par les philologues, on retrouve les catalogues dans le débat sur le rapport entre Homère et Hésiode, sur la datation des deux poètes « originels », sur la localisation géographique de la poésie catalogique, sur la classification problématique de la matière catalogique au sein de l'œuvre homérique, sur l'évaluation de l'historicité d'Homère et de son rapport à l'époque mycénienne et la forme inventoriale ${ }^{1}$. Sous cette même rubrique de l'influence et de la transmission, on peut classer le débat qui associe la naissance de l'histoire à l'hypothèse de l'existence de chroniques prélittéraires, développées à partir de l'annotation

\footnotetext{
${ }^{1}$ À titre purement indicatif, quelques étapes bibliographiques: M.L. WeST, The Hesiodic Catalogue of Women, Oxford, 1985; J. ButTERWORTH, " Homer and Hesiod », in Studies in honor of T.B.L. Webster I, Bristol, 1986, p. 33-45; O. DiCKINSON, "The Catalogue of Ships and all that », in Meletemata I, in honour of M.H. Wiener, Liège, 1999, p. 209-210; M. EDWARDs, « The Structure of Homeric Catalogues », in TAPhA 110 (1980), p. 81-105.
} 
des listes officielles ${ }^{2}$, ainsi que le statut problématique de « la popularité de la poésie généalogique d'Hésiode parmi les premiers historiens $»^{3}$. Le comparatisme interculturel s'interroge de son côté sur l'influence de la pratique des listes orientales sur l'utilisation de la forme catalogique en Grècé ; or, comme en témoigne le débat autour des thèses de Goody, divers facteurs rendent ce rapprochement problématique.

La liste incomplète des questions ouvertes relevées ci-dessus renvoie in fine au "Grand partage ». Goody associe la pratique mésopotamienne des listes à la naissance de l'histoire et y décèle un processus notoire d'abstraction, rendu possible par l'écriture. Dans la même logique, le cas grec du passage du muthos à l'histoire résiderait dans la juxtaposition hécatéenne des logoi par l'écriture, qui rendrait les contradictions évidentes. 5 . Fr. Hartog a mis en évidence un certain nombre de spécificités grecques qui affaiblissent le rapport entre liste, écriture et naissance de l'histoire. Le cas des généalogies démontre le flottement durable entre historia et genealogia, malgré la pratique de l'écriture ${ }^{7}$. En attendant de réelles avancées dans le domaine comparatiste, le schéma de Goody reste une hypothèse dont l'intérêt n'est pas épuisé.

Un autre point fort des travaux de Goody, plutôt méconnu des hellénistes, est la mise en évidence de l'interêt philosophique (épistémologique) des listes en tant que mode de classement. Pour employer son vocabulaire, ce processus «artificiel» qu'est la constitution des listes exprime le début de l'évolution qui conduira à la logique formelle d'Aristote : « La liste implique discontinuité et non continuité »... Les listes diffèrent beaucoup des formes de langage parlé, parce qu'elles en disjoignent les éléments et les traitent abstraitement. Le fait même de construire des tableaux simples... peut amener à se poser des questions concernant les oppositions ou les contrastes, les analogies et les contradictions... et contribuent à la constitution d'une « logique formelle $»^{8}$. En Grèce ancienne, l'importance de ce mode logique particulier

\footnotetext{
${ }^{2}$ F. JACOBY, Atthis. The Local Chronicles of Ancient Athens, Oxford, 1949, p. 176 sq. (avec les références bibliographiques antérieures) et infra.

3 L. BerTELli, "Hecateaus, From Genealogy to Historiography », in N. LuRAGHI (éd.), The Historian's Craft in the Age of Herodotus, Oxford, 2001, p. 73.

${ }^{4}$ WEST, o.c. (n. 1), p. 11 sq. Sur les interférences entre Grèce et Orient, voir J. GOODY, La raison graphique, Paris, 1979, ch. VII; Entre oralité et écriture, Paris, 1994, 2 e partie; L'bomme, l'écriture et la mort, Paris, 1996, ch. VIII.

${ }^{5}$ Voir J. GOODY, La raison graphique, Paris, 1979, et pour le débat helléniste, M. DETIENNE, L'invention de la mythologie, Paris, 1981, p. 194 sq.; F. HARTOG, Évidence de l'Histoire. Ce que voient les historiens, Paris, 2005, p. 45-55; R. THOMAs, Literacy and Orality in Ancient Greece, Cambridge, 1993, p. 16 sq.; BerTelli, l.c. (n. 3), p. 67-72; Cl. CALAME, ici même.

${ }^{6}$ Qu'Hartog traite, en l'occurence, comme un exemple de liste (voir infra). De même R. Thomas, Oral Tradition and written Record in Classical Athens, Cambridge, 1989, p. 180.

HARTOG, o.c. (n. 5), p. 49. Sur la généalogie comme « espace intellectuel de comparaison et de critique », Chr. JACOB, «L'ordre généalogique entre le mythe et l'histoire », in M. DETIENNE (éd), Transcrire les mythologies, Paris, 1994, p. 182.

${ }^{8}$ GOODY, o.c. (n. 5), p. 182. La fonction inventoriale - économique - n'est pas discutée dans le présent article.
} 
se manifeste dans son réemploi intergénérique ininterrompu à travers les siècles. Comme le dit Lambros Couloubaritsis, du catalogue au syllogisme, l'histoire de la pensée grecque peut se dire par les composés de $\lambda \hat{\gamma} \gamma \omega$, de $\varkappa \alpha \tau \alpha \lambda \varepsilon_{\gamma} \omega$ à $\delta\llcorner\alpha \lambda \varepsilon ́ \gamma o \mu \alpha !$ jusqu'à la définition des syllogismes chez Aristote.

En effet, L. Couloubaritsis montre que le point de vue philosophique est d'une importance majeure pour comprendre la forme catalogique en tant que discours qui doit être « décodé et redressé pour manifester le réel dans sa structure globale ${ }^{10}$. Pour exprimer son objet selon des ordres de succession divers, la forme catalogique se sert de schèmes (dans le sens kantien) fondés sur l'expérience réelle proche, d'où la nécessité de redressement. En témoigne fortement la généalogie, que j'envisage comme un sous-genre de la forme catalogique. La généalogie utilise le schème le plus familier à l'homme, le schème de la parenté, qui articule le sensible à l'invisible aussi bien dans les « catalogues des ancêtres » que dans les théogonies. Cette articulation entre deux ordres différents, effectuée par le lien de la parenté, donne aux relations généalogiques leurs potentialités conceptuelles, comme le dira Socrate dans Théetète $(155 \mathrm{~d})^{11}$.

D'autres schèmes produisent divers types de catalogues, le schème du chemin, de la quête, se traduira par un catalogue géographique, voire de topologie mythique ou de multiples voyages. Dans ce langage, le katalegein renvoie à une pratique du logos, celle de former des ensembles discrets, ce qui permet d'envisager le passage du muthos au logos comme «passage d'une pratique du logos à une autre pratique du logos, donc aussi passage d'un type de rationalité à un autre type de rationalité ». La conception philosophique de la pratique du katalegein est décisive pour faire la part entre les analyses qui accordent un statut épistemologique à la forme catalogique ( $\mathrm{T}$. Krischer) et celles qui y voient surtout une «poétique inventoriale » $(\mathrm{S} \text {. Perceau })^{12}$, une structure descriptive ouverte et extensible à l'infini. Il en va, en fait, de l'interprétation globale de la pensée archaïque soit

\footnotetext{
${ }^{9}$ Voir ici-même. Voir aussi L. COUlOUBARITSIS, Conférence sur le mythe (24 avril 2001), Académie d'Athènes, Bulletin de l'Académie d'Athènes 76 (2) (2001), p. 322-340 (en grec moderne).

10 «Transfigurations du paradigme de la parenté », in J. JAYON et J.J. WunENBurger (éds), Le paradigme de la filiation, Paris, 1995, p. 175.

${ }^{11}$ Généalogie d'Iris, fille de Thaumas. Thomas, o.c. (n. 5), p. 175-177; voir aussi Théetète, 152d. L'expression " catalogue des ancêtres » est de Platon (ibid., 175a). Sur l'extension de la généalogie comme mode d'articulation conceptuelle de l'expérience humaine, L. CouloubaRITSIS, Histoire de la philosophie ancienne et médiévale, Paris, 1998, I ${ }^{\mathrm{re}}$ partie.

${ }^{12}$ T. KRISCHER, Formale Konventionen des Homerischen Epik, Munich, 1971 (Zetemata, 56); S. PerceAu, La parole vive. Communiquer en catalogue dans l'épopée homérique, Louvain, 2002, dont le livre riche est, par certains aspects, encore tributaire d'une lecture évolutionniste d'Homère; par ailleurs, il me paraît impossible de dissocier l'étude du katalegein de l'option narrative plus générale de l'école homérique face à celle hésiodique (l'espace ne permet pas d'inclure ici le volet de mon travail spécifiquement consacré à Homère, présenté au S.I.R.E en plusieurs séances sous le titre Katalegein, mutheuesthai, poiein, ULB, mai - juin 2004).
} 
dans le sens évolutionniste du primitivisme ou d'une manipulation consciente et refléchie de la distorsion mythique ${ }^{13}$.

\section{L'Histoire en train de se faire}

«L'auteur est aussi un lecteur... il n'existe pas d'inventions génériques ex nibilo, mais seulement des réaménagements, amalgames ou extensions à partir d'horizons génériques déjà disponibles »" ${ }^{14}$. L'idée qui motive le présent article est que l'usage de la forme catalogique et de la généalogie dans l'historiographie inscrit les auteurs dans une filiation intellectuelle. Hérodote sera l'exemple privilégié, examiné à partir de la configuration des « origines » de la poétique grecque - Hésiode et Homère - qui présente deux options, une école " catalogique » hésiodique face à une école homérique qui instaure un mode mixte à dominance «mimétique », « dia-logique ». Comme le montrent de façon différente Platon et Aristote, la forme dialogique et la prédominance du discours direct sont consubstantiels à Homère et la Poétique d'Aristote témoigne de l'importance de cet apport pour l'histoire de la formation des genres, donc de la pensée grecque ${ }^{15}$.

Occupant une position de princeps, «à jamais énigmatique » ${ }^{16}$, Hérodote use amplement des ressorts de la mimésis et de l'émotion. En témoigne la structure des épisodes qui encadrent le grand catalogue des forces de Xerxès, marqué, sur le plan de "l'intrigue »", par l'émotion de Xerxès et l'étonnement des spectateurs internes (infra). L'adoption plus systématique de l'option narrative homérique dans les livres VII à IX (particulièrement représentatifs du style « unique » d'Hérodote ${ }^{18}$ ), s'explique par un changement de perspective : dans les livres de la guerre, la représentation des faits l'emporte sur l'autopsie,

13 COUlOubaritsis, l.c. (n. 10), p. 175 sq.; l.c. (n. 11), p. 33-64 et La Proximité et la question de la souffrance humaine, Bruxelles, 2005, p. 70. Sur les catalogues généalogiques voir infra.

${ }_{15}^{14}$ J.-M. SCHAEFFER, Qu'est-ce un genre littéraire?, Paris, 1989, p. 153.

${ }^{15}$ Voir, récemment, R. Martin : «The genius of the homeric composers is to vacuum up the subgenres that naturally occur on their own in the oral poetic surroundings and put them to new and pointed use » (in R. HunTER, The Hesiodic Catalogue of Women, Cambridge, 2005, p. 172, livre intéressant pour réfléchir sur les transformations diachroniques de la forme catalogique). I. RUTHERFORD, «Formulas, Voice, and Death in Ehoie-Poetry, the Hesiodic Gunaikon Katalogos, and the Odysseian Nekuia », in M. DEPEW et D. OBBINK (éds), Matrices of Genre. Authors, Canons and Society, Cambridge MA., 2000, p. 87 : «Absence of direct speeches in general was regarded as typical of the genre... »

${ }^{16}$ E. BAKKer, I. DE JONG, H. VAN WeEs (éds), Brill's Companion to Herodotus, Leiden, 2002, p. xvii et HARTOG, o.c. (n. 5), p. 45-61.

${ }^{17}$ Sur cette notion appliquée à l'œuvre d'Hérodote en rapport avec le mot logos, P. PAYEN, Les îles nomades, Paris, 1997, p. 63 sq. Voir aussi id., «Historia et intrigue. Les ressources mimétiques de l'Enquête d'Hérodote », à paraître dans les DHA.

18 J. MARINCOLA, «Herodotean Narrative and the Narrators' presence », Arethusa 20 (1987), p. 131, insiste là-dessus : «Even if we recognize that Persika were written before... it is imperative to realize that Herodotus' presentation was different, and his narrative unique. " Sur les Persika avant Hérodote, R. DREws, The Greek Accounts of Eastern History, Cambridge Mass., 1973. 
le « je » du narrateur se fait donc plus rare pour laisser la place à la «visualisation » des événements et de leurs acteurs ${ }^{19}$.

Les deux options poétiques originelles semblent avoir pesé considérablement sur le lent processus de la formation des genres, l'historiographie n'étant pas une exception. On peut, en effet, considérer que la question de la forme catalogique, même si elle n'est pas formulée comme telle, fait partie du débat sur la naissance de l'histoire, en dehors de la théorie de Goody, puisque la critique de Jacoby contre Wilamowitz à propos des sources et de la forme des premières œuvres « historiographiques », notamment à propos de l'existence des chroniques « annalistiques » locales avant Hérodote, a dominé l'interprétation des historiens ${ }^{20}$. Au-delà de ce débat, toujours ouvert, il est important de mettre en relief cette autre source d'influence sur l'écriture historiographique en catalogue qu'est la poésie ${ }^{21}$, puisque des auteurs anciens témoignent en ce sens ${ }^{22}$.

Le témoignage crucial à ce propos, très controversé parce qu'il contredit la position radicale de Jacoby ${ }^{23}$, est celui de Denys d'Halicarnasse, qui donne, dans son De Thucydide (VII, 5) un catalogue des auteurs qui ont précédé Thucydide et dont le style est décrit de façon unitaire : «langage clair, courant, pur, concis, adapté au sujet, qui ne laissait soupçonner aucun procédé de mise en œuvre ». "Ils n’ajoutent ni ne retranchent rien », ( $\mu$ ríte

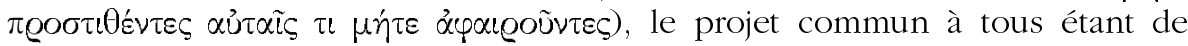
«transmettre tels qu'ils l'ont reçu (oí $\alpha \varsigma \pi \alpha \varrho \varepsilon ́ \lambda \alpha \beta o v)$ la mémoire conservée par les locaux, dans les cités et les pays, et s'il y avait dans des lieux profanes ou

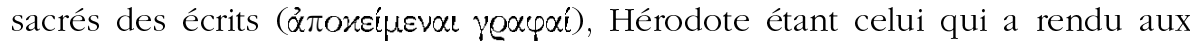
style historiographique les qualités négligées par les autres ( $\tau \dot{\alpha} \varsigma \pi \propto \varrho \alpha \lambda \varepsilon \vee \varphi-$

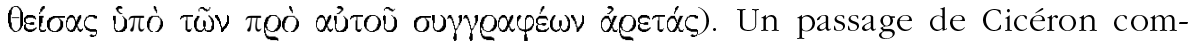

\footnotetext{
${ }^{19}$ MARINCOLA, l.c. (n. 18), p. 132. La question de savoir si l'influence provient d'Homère ou du drame attique est un faux dilemme, du moment où ce dernier est une application du modèle homérique. Sur Hérodote et Homère : K. WATERS, Herodotus the Historian, Oklahoma, 1985, p. 61 $s q$. Voir aussi la nouvelle préface de Fr. HARTOG, Miroir d'Hérodote, Paris, 2002. Sur la voix des autres chez Hérodote, C. DARBo-Peschanski, Le discours du particulier, Paris, 1987, p. 120-126. Sur la question du passage de la poésie à la prose, voir récemment, BerTeldi, l.c. (n. 3) et Cl. CALAME, ici-même.

${ }^{20}$ Bref aperçu et bibliographie chez BerTelli, l.c. (n. 3), p. 70-72; et aussi, ibid., A. Moller, «The Beginning of Chronography: Hellanicus' Hiereiai» et l'article important de R. FOwLER, «Herodotus and his contemporaries », JHS 116 (1996), p. 62-87. Voir aussi P. PAYEN, " L'historiographie grecque : vI ${ }^{e}-$ III $^{e}$ siècle avant J.-C. (jusqu'à Phylarque) », Pallas 63 (2003), p. 129-166 (bibliographie critique).

${ }^{21}$ Concernant la question intéressante posée par A. MOLLer (ibid.), il me semble difficile de nier l'influence des généalogies sur le pattern annalistique, chez Hellanikos par exemple: les généalogies ne mesurent certes pas le temps comme les annales, mais fournissent un modèle catalogique d'ordonnancement du récit qui se transforme aisément en annalistique.

${ }^{22}$ Voir D. TOYE, «Dionysius of Halicarnassus on the first Greek Historians », AJPh 116 (1995), 279-302.

${ }^{23}$ Jacoby, o.c. (n. 2), p. 178 sq.; A. Momigliano, Studies in Historiography, Londres, 1966, p. 127 sq.; C. FORNARA, The Nature of History in Ancient Greece and Rome, Berkeley, 1983, p. 17 sq.; FOWLER, l.c. (n. 20), p. 62 sq.
} 
plète d'habitude ${ }^{24}$ la lecture de Denys : contrairement à Hérodote, Phérécyde, Hellanikos, Akousilaos « et beaucoup d'autres parmi les Grecs» ont adopté un style d'écriture proche des annales maximi (De oratore, 2, 53 et 55), style par excellence catalogique. Clément d'Alexandrie reprochait à Eumelos et à Akousilaos d'Argos, auteurs tous les deux d'œuvres généalogiques, de ne rien avoir fait d'autre que de mettre Hésiode en prose $e^{25}$. Flavius Josèphe exprime la même idée avec plus de précision ${ }^{26}$ : Hellanikos de Lesbos a corrigé Akousilaos qui a corrigé Hésiode (FGrHist 2 T 6). Pour les Alexandrins, le style catalogique "pur » porte le sceau d'Hésiode, et c'est sur cet argument stylistique qu'un catalogue énumératif sec chez Homère peut être athétisé par Zénodote et Aristarque ${ }^{27}$.

Ce texte de Denys n'a pas intéressé Jacoby, puisqu'il se fonde sur le critère du style ${ }^{28}$. Raisonnant en évolutionniste, Denys considère que le style simple a dû précéder Hérodote ${ }^{29}$. Il n'est pourtant pas nécessaire - ni possible, actuellement - de poser les deux styles d'écriture en rapport chronologique, surtout si on admet l'hypothèse d'une influence décisive de la "dichotomie " originelle de la poétique grecque sur la naissance de l'histoire, notamment à cause des liens entre histoire et généalogie et de l'innovation hérodotéenne comme contre-proposition "historique » sur le plan aussi bien de la forme que du contenu.

Toute une ligne de lecteurs, qui remonte au moins à Théophraste ${ }^{30}$, semble tracer une ligne de démarcation fondamentale dans le courant historiographique entre des exposés secs et factuels, plus proches de la structure catalogique, et la valeur littéraire de l'œuvre d'Hérodote - l'admirateur d'Homère, comme l'appelle Denys, ou "le très homérique », comme l'appelle Longin qui lui confère un statut particulier dans le métier historiographique : princeps genus hoc ornavit (Cicéron, De oratore, 2, 55). Entre ces deux façons de faire l'histoire, qui entretiennent un rapport différent à la poésie, la tension durera : Polybe, au II ${ }^{\mathrm{e}}$ siècle av. J.-C., stigmatise les historiens qui mêlent le pathos à leur composition ${ }^{31}$. Le critère de la vérité n'est pas l'unique fondement de l'histoire tout au long d'une tradition de réception et la tension entre historiens et littéraires dans les études hérodotéennes traduit en partie ce débat.

\footnotetext{
${ }^{24}$ Voir FORNARA, O.c. (n. 23), p. 19; F. LASSERRE, «L'historiographie grecque à l'époque archaïque », QS 4 (1976), p. 112-114.

25 Clément d'Alexandrie, Stromates VI, 26, 7. Je rappelle que dans le présent article les généalogies sont envisagées comme des sous-genres de la forme catalogique.

${ }^{26}$ Comme le remarque FORNARA, o.c. (n. 23), p. 4.

${ }^{27}$ Scholie à Il. XVIII, 39-49. MARTIN, l.c. (n. 15), p. 171. Voir aussi Gr. NAGY, « Aristarchean questions », Bryn Mawr Classical Review 98.7.14.

${ }^{28}$ FOWLER, l.c. (n. 20), p. 66 : « style is no very bad criterion -indeed, it is a better one than Jacoby's, if you have nothing else to go on. »

${ }^{29}$ Comme le note FORNARA, o.c. (n. 23), p. 19.

${ }^{30}$ Cicéron, De oratore, 12, 39 avec les précisions de LASSERRE, l.c. (n. 24), p. 113-114.

${ }^{31}$ Polybe (XII, 26, 9) et Plutarque critiquent Phylarchos qui accompagne d'ornements dramatiques le récit des événements (fr. 81 [éd. F. JACOBY]); FORNARA, o.c. (n. 23), p. 61 sq.
} 


\section{Comment gérer (de façon originale) un grand catalogue}

Avant Hérodote et autour de lui, il y a déjà une tradition importante de catalogues. Lui-même s'y emploie couramment. Le verbe katalegein n'a pas un sens métaphorique dans son discours : 36 occurrences dont 28 sont suivies d'un vrai catalogue. « Chez Hérodote le verbe katalegein prend toujours le sens d'énumérer et décrire $»^{32}$. Au tout début de son œuvre apparâit déjà le premier catalogue (I, 6) : dans l'effort d'élucider la question du premier responsable de la guerre de Troie, il «parcourra » et « se souviendra » de « petites et des grandes cités des hommes ». Ce premier « parcours » catalogique est une généalogie qui met en marche le temps intra-textuel ${ }^{33}$ et qui situe les conquérants dans leur espace géographique et les réunit dans le même inventaire selon un critère précis, leur rapport aux Grecs d'Asie Mineure.

Qu'Hérodote ait appliqué en prose le modèle homérique ${ }^{34}$ est une constatation ancienne. Or, actuellement, ses affinités génériques soulèvent encore un âpre débat. Entre l'« historiopoï̀te » et le «premier présocratique entièrement conservé $»^{35}$, ouvert aux progrès scientifiques et philosophiques de son temps, l'articulation est loin d'être réglée ${ }^{36}$. Pourtant, examiner l'homérisation consciente et flagrante de l'Enquête ne revient pas à nier le faisceau des différents courants de pensée qui la nourissent, mais à s'interroger sur un trait intergénérique dans la pensée grecque, c'est-à-dire le rôle décisif des poètes originels dans la formation des genres discursifs majeurs.

Deux passages de l'œuvre, très discutés, me serviront de terrain d'observation : l'épisode d'Hécatée récitant sa généalogie en Égypte et le long inventaire des contingents de l'armée de Xerxès (livre VII, 61-99), rassemblée à Doriscos en vue de l'expédition contre la Grèce, que je vais commenter en premier, parce qu'il illustre mieux que tout autre «le travail de déplacement et de transformation $»^{37}$ qu'Hérodote opère sur cette forme très ancienne qu'est le catalogue. Un même mouvement d'«homérisation » définit ce pas-

\footnotetext{
${ }^{32}$ Comme le remarque PAYEN, o.c. (n. 17), p. 100. E. Powell, Lexicon to Herodotus, Cambridge, 1938 , s.v. katalegein

33 I. DE JONG, « Narrative Unity and Units », in BAKKER et al., o.c. (n. 16), p. 253.

${ }^{34}$ H. ERBSE, Studiens zum Vertändnis Herodots, Berlin / New York, 1992, p. 125-127 (sur Homère et le catalogue de Xerxès); Gr. NAGY, Pindar's Homer, Baltimore, 1990, p. 231-233; récemment, D. BOEDEKER, «Epic Heritage and mythical patterns in Herodotus », in BAKKER et al., o.c. (n. 16), p. 99-115, et DE JONG, l.c. (n. 33), p. 245-266.

35 J. MYRES, Herodotus Father of History, Oxford, 1953, p. 43.

${ }^{36}$ Comme le rappelle encore récemment R. THomas, Herodotus in context, Cambridge, 2000, p. 9; son désaccord avec Gr. Nagy en est une démonstration (voir, à ce propos, E. BAKKER, « The Making of History: Herodotus' Historiês Apodexis », in BAKKER et al., o.c. [n. 16], p. 3-32). Sur le rapport entre histoire et autres genres poétiques, voir E. BowIE, « Ancestors of Historiography in Early Greek Elegiac and Iambic Poetry? », in LuRAGHI, o.c. (n. 3), p. 45-66.

${ }^{37}$ PAYEN, O.C. (n. 17), p. 102.
} 
sage ainsi que l'ensemble de la séquence dans laquelle il s'inscrit et dont la structure me semble relever de l'ainos tel que Gr. Nagy l'a analysé ${ }^{38}$.

Dans la symbolique de l'Enquête, qui, depuis le préambule, se place sous le signe épique ${ }^{39}$, la mise en scène de la progression des forces de Xerxès vers l'Europe renvoie très concrètement à l'affrontement iliadique entre Grecs et Asiates. Dans la première énumération (VII, 41), avant la traversée de l'Hellespont, qui décrit uniquement l'armée d'élite perse autour de Xerxès, le premier arrêt mentionné a lieu à la plaine de Troie. Sont nommés les lieux symboliques de l'Iliade, le Scamandre, la citadelle de Priam, Athéna Ilias à laquelle Xerxès offre un sacrifice de mille boufs et "les héros » que les mages honorent par des libations (VII, 43). Ces gestes intertextuels sont fortement dramatisés puisqu'ils dépendent de l'émotion de Xerxès : c'est le bimeros qui le pousse à ce pèlerinage. La mention d'Athéna Ilias renvoie subtilement au sort tragique d'Hector auquel la déesse n'a pas accordé la victoire malgré la riche offrande du peplos.

Un long développement catalogique chez Hérodote est toujours motivé car la fonction inventoriale ne constitue pas la dominante de l'œuvre ${ }^{40}$. De toute façon, la longueur excessive appelle des explications : « je prolonge le logos sur les Égyptiens, car ils ont pleista thaumasia (II, 35) »" La mise en scène du Catalogue des forces relève de la même logique, renforcée par sa facture homérique : arrivé à Abydos, juste avant la traversée de l'Hellespont, Xerxès souhaite contempler l'ensemble de son armée (VII, 44, 1-2), à partir d'un lieu haut ${ }^{42}$. Le spectacle l'émeut: d'abord il le remplit de joie (i $\mu \varepsilon ́ \varrho O r$.

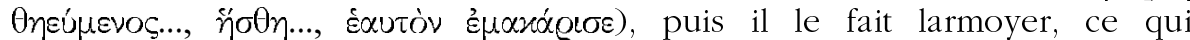
provoque le commentaire bien connu de son oncle Artabanos sur les dangers stratégiques du grand nombre et sur la fragilité du sort humain ${ }^{43}$. Puis, une fois la traversée interminable achevée, qui a duré sept jours et sept nuits, Xerxes choisit la plaine de Doriscos pour rassembler l'armée, au bord de la mer. Hérodote tient à décrire ce que voit Xerxès dans toute son étendue, parce que c'est un événement d'envergure exceptionnelle.

\footnotetext{
${ }^{38}$ La structure narrative du catalogue s'éclaire par les travaux de Gr. NAGY sur Hérodote et l'ainos : "Mythe et prose en Grèce archaïque : l'ainos », in C. CALAmE (éd), Métamorphoses du mythe en Grèce ancienne, Genève, 1998, p. 229-242 et Pindar's Homer, cit. (n. 34), p. 248-249.

39 Tout comme la fin des Histoires, qui terminent avec un signe du monde épique vers le monde contemporain : la vengeance de Protesilaos, voir Gr. NAGY, « The Sign of Protesilaos », Metis 2 (1987), p. 207-213

${ }^{40}$ PAYEN, o.c. (n. 17), p. 101. La longeur d'un discours chez Homère correspond exactement au statut du personnage : R. MARTIN, The language of Heroes, Ithaca, 1989, p. 46.

${ }^{41}$ Voir aussi III, 60 et C. DEWALD, «Epic Heritage and Mythical Patterns in Herodotus », in BAKKER et al., o.c. (n. 16), p. 282.

${ }^{42}$ Xerxès fait la même chose que Darius au Bosphore (IV, 85).

${ }^{43}$ Sur la nature tragique de l'épisode, WATERs, o.c. (n. 19), p. 68 sq. Plus généralement S. SAÏD, « Herodotus and Tragedy », in BAKKER et al., o.c. (n. 16), p. 117-147.
} 
La mise en œuvre visuelle du catalogue des forces, qui se déroule sous le signe du spectacle ( $\theta \varepsilon \tilde{\omega} \mu \alpha$ ! marquant le début et la fin de ce long récit $^{44}$ ), constitue une autre trace importante de transposition de la technique homérique, qui consiste à manipuler la forme catalogique pour produire un effet visuel (articuler donc le catalogique au visuel) ${ }^{45}$. Homère décrit les meilleurs de l'armée achéenne à travers le regard d'Hélène, du haut des murailles troyennes dans la Teichoskopia. Un autre exemple de cette adaptation : le catalogue des femmes du chant XI de l'Odyssée est " performé » par Ulysse et

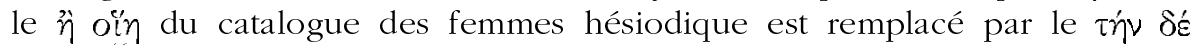
¿'óv... Tout comme le cas d'Hélène dans l'Iliade, dont l'œil organise le catalogue des chefs, cet expédient narratif sert à accentuer le rôle de Xerxès dans ce grand mouvement humain. C'est ainsi que le catalogue devient un élément «argumentatif » de type particulier, soumis à la forme codée de l'ainos, puisque sa justification narrative se fait retrospectivement, à partir de la discussion entre Xerxès et Démarate, qui préfigure l'issue ultérieure de cette expédition monumentale.

Ces techniques de dramatisation semblent reposer sur la surdétermination iliadique de Xerxès - Xerxès-Hector, Xerxès-Agamemnon. L'homérisation du récit signifie que la narration atteint un point particulièrement crucial, le moment où l'étroit passage qui sépare les deux continents sera franchi ${ }^{47}$. Que l'Hellesponte fonctionne comme signe fort d'homérisation, la fin de l'Enquête le démontre amplément, toujours sur le mode particulier de l'ainos : dans les paroles d'Artuaktès, qui veut posséder «l'oikos » (le tombeau) de Protesilaos et ses richesses, le héros homérique mériterait une telle « dépossession » parce que, répète-t-il deux fois, il a " attaqué la terre de Xerxès... »" ${ }^{48}$

Comme pour le Catalogue des Vaisseaux iliadique $^{49}$ un récit de rêves prémonitoires précède l'énumération des forces de Xerxès ${ }^{50}$. Aussi, Hérodote ouvre la partie proprement énumérative de son catalogue par une phrase qui

\footnotetext{
${ }^{44}$ Aecoual : VII, 56; 100, 3 \& 15. Voir D. KOnSTAN, «Persians, Greeks and Emprire » et la réponse de J.-P. Vernant dans Arethusa, l.c. (n. 18), p. 59-82.

${ }^{45}$ Voir déjà Cicéron, Disputationes Tusculanae IV 114. Sur la mise en scène du taxinomique (« l'œil comme point focal ») : F. HARTOG, Le miroir d’Hérodote, Paris, 1991, p. 262. Le rapport entre Hérodote et le théâtre (par ex., comment le mode mimétique peut « animer » un catalogue, comme dans le Promethée enchaîné, 705 sq.) mérite une discussion à part; voir, en général, SAÏD, l.c. (n. 43); Drews, o.c. (n. 18), p. 32 sq.

${ }^{46}$ RUTHERFORD, l.c. (n. 15), p. 93.

${ }^{47}$ H.R. IMMERWAHR, Form and Thought in Herodotus, Ohio, 1966, sur l'importance symbolique de ces frontières dans l'œuvre d'Hérodote.

48 'I'ñv ờv yñ (IX, 116, 3), dit Artuaktès en s'adressant à Xerxès : NAGY, Pindar's Homer, cit. (n. 34), p. 268-273

${ }^{49}$ Voir récemment BOEDEKER, l.c. (n. 34), p. 103. Sur la maîtrise des techniques épiques par Hérodote, P. PuccI, «L'apologie d'Apollon dans Hérodote 1, 91 », Metis 8 (1993), p. 13-14.

${ }^{50}$ Cf. Iliade, II, 7-34, le rêve trompeur que Zeus envoie à Agamemnon; le rêve de Xerxès : VII, 12-18. À propos de ce rapprochement voir les réserves de SAÏD, l.c. (n. 43), p. 142 sq; O. MurRay, "Herodotus and Oral History », in LuRAGHi, o.c. (n. 3), p. 33 : rêve et message moralisant : « Herodotus' own creation. »
} 


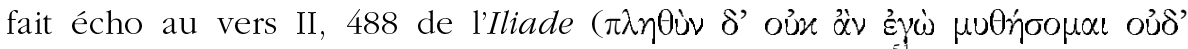

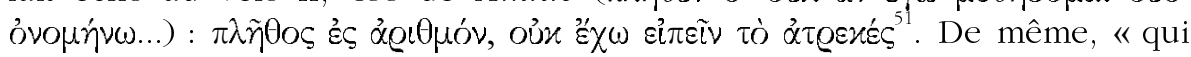
pourrait nommer tous ceux qui viennent animer le combat» autour du cadavre de Patrocle (XVII, 260-261) ? Difficile de «tout nommer », l'exhausti-

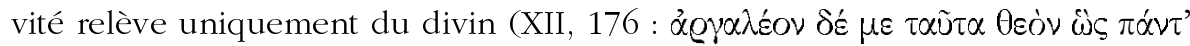

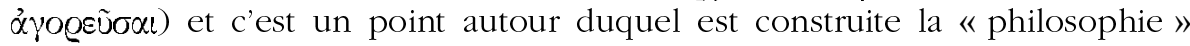
homérique des catalogues (voir infra).

«Les Grecs sont moins les inventeurs de l'histoire que de l'historien comme sujet écrivant $»^{52}$. Tout en empruntant à Homère le trope de l'invocation conventionnelle qui précède un catalogue, Hérodote substitue aux Muses la parole contemporaine des logoi: il ne pourra pas donner les chiffres par contingent (voir aussi 54,3), puisque aucun peuple ne conserve des logoi à

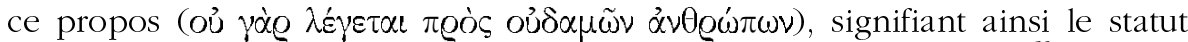
propre à son ouvre, qui ne prétend pas à l'inspiration surnaturelle ${ }^{53}$. Mais, si on tient compte de la dimension polémique et agonistique du « je » hérodotéen, l'intertextualité tissée dans le logos d'Hérodote pourrait être aussi un signe d'émulation ${ }^{54}$.

Ou legetai fait penser inévitablement au débat crucial autour des sources orales ou écrites d'Hérodote dans ses étapes de F. Jacoby à O. Murray, dont le «documentary model ${ }^{55}$ assure le relais entre les documents achéménides et l'information donnée par Hérodote. Murray parvient à déplacer le débat parce qu'il tient compte de la perspective aussi bien littéraire qu'historienne : Hérodote n'entretient pas un rapport statique avec les sources, mais réorganise les testimonia, selon la perspective originale, propre à son projet esthétique et moralisant $^{56}$. Ce qui explique l'usage plus «argumentatif » que purement énumératif et informatif des catalogues qu'Hérodote choisit de déployer ${ }^{57}$.

\section{Les forces de Xerxès et les richesses de Crésus}

P. Payen a commenté brièvement mais de façon substantielle les limites de l'influence épique en matière de catalogues et le caractère conscient de la manipulation hérodotéenne. Indéniablement, le catalogue des forces de Xerxès a également une visée ethnographique : associé à celui des satrapies (III, $90 s q$.) et à la description de la voie royale (V, 52 sq.), il participe à la

\footnotetext{
${ }^{51}$ Sur la atrekeia, DARBO-PESChanski, o.c. (n. 19), p. 179 sq.; P. CARTLEDGE, E. GReENWOOD, « Herodotus as a Critic: Truth, Fiction, Polarity », in BAKKER et al., o.c. (n. 16), p. 361-363.

${ }^{52}$ HARTOG, O.c. (n. 5), p. 46, citant Wilamowitz.

53 C. CAlame, Le récit en Grèce ancienne, Paris, 2000, p. 128-130; Thomas, Literacy and Orality, cit. (n. 5), p. 114-116; PuccI, l.c. (n. 49), p. 13-15.

54 Sur les dimensions polémique et agonistique du « je » hérodotéen, voir Marincola, l.c. (n. 18); BOEDEKer, l.c. (n. 34); P. PUcCI, Odysseus polytropos, Lille, 1995 (trad. fr).

${ }^{55}$ Avec les nuances de N. LURAGHI, «Introduction », in LuRAGHI, o.c. (n. 3), p. 10 sq.

${ }^{56}$ MurRay, l.c. (n. 50), p. 28.

${ }^{57}$ L'usage argumentatif des catalogues est clairement énoncé par Hérodote lui-même, à propos des prestations catalogiques d'Artabanos aux côtés de Darius (IV, 83, 2) et d'Hécatée aux côtés d'Aristagoras (V, 36, 2).
} 
description de l'Empire ${ }^{58}$. L'activité détaillée d'énumération et d'agencement des contingents (VII, 60 sq.) que Xerxès commande après la traversée, est présentée en structure annulaire qui souligne la composante visuelle ${ }^{59}$. Jusqu'en VII, 83, Hérodote décrit les contingents, leur uniforme et armement, nomme leur chef, précise la façon de diviser ces contingents et d'attribuer des officiers à chaque entité (VII, 81) ${ }^{60}$. Suit la mention des chevaux, autre écho iliadique (VII, 84-88, Iliade II, 761 sq.). Et du paragraphe 89 au paragraphe 100, Hérodote construit un Catalogue des Vaisseaux.

Quand Armayor relevait qu'il y a 67 nations dans l'empire de Darius, 67 contingents dans l'armée de Xerxès, comme il y a 67 chefs, achéens et troyens réunis, dans le Catalogue des Vaisseaux iliadique, il concluait qu'il fallait inscrire le catalogue que nous examinons dans la longue tradition littéraire du même type. Les chiffres qu'Hérodote avance en VII, 186-187 sont invraisemblables, selon les spécialistes. En revanche, même s'il ne s'agit pas d'une « liste », dans le sens d'une transcription d'un document officiel, ce catalogue est peut-être le seul cas, selon D. Lewis, où il vaut la peine d'essayer de départager noyau «documentaire » et ce qui relève de la transformation littéraire ${ }^{61}$.

La fonction polyvalente, « historiopoḯtique » ${ }^{62}$, du catalogue se laisse lire à partir de l'échange entre Xerxes et Démarate, Artabanos et Xerxès dans le contexte immédiat, mais aussi à partir des dialogues entre Solon et Crésus. Hérodote met en scène les options stratégiques de Xerxès en en indiquant l'extravagance inquiétante par la simple longeur de l'énumération déployée dans l'espace du récit. L'ambiguité stratégique du critère arithmétique, prédominant dans les décisions de Xerxès, est déjà esquissée dès après la premier « spectacle » catalogique, dans sa discussion avec Artabanos (VII, 47-48), où Xerxès, croyant qu'Artabanos doute de l'expédition à cause du nombre insuffisant des forces perses, propose de les augmenter et Artabanos le prévient que le nombre exorbitant peut se retourner contre le Grand Roi ${ }^{63}$.

Si le grand nombre n'est pas résumé ici en quelques phrases ${ }^{64}$, c'est aussi pour renforcer la position éthique hérodotéenne face à la richesse que l'on

\footnotetext{
${ }^{58}$ W.W. How, J. Wells, Commentary on Herodotus, Oxford, 1961, II, p. 151-152.

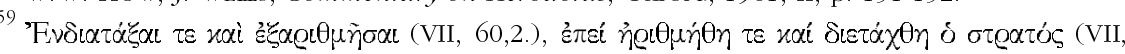
100). PAYEN, o.c. (n. 17), p. 103 : «Les mêmes mots ou des termes construits sur les mêmes radicaux présents à huit reprises dans les chapitres 59-60. »

${ }^{60}$ En nuançant le rapprochement, P. PAYEN (ibid., p. 100) fait remarquer qu'Hérodote met au centre les peuples et leurs coutumes et pas les chefs.

${ }^{61}$ D. LEWIS, Selected papers in Greek and Near Eastern History, Cambridge, 1997, p. 345-361. Sur la notion de « document pur » et les pièges du " positivisme sémantique », voir N. LORAUX, " Thucydide n'est pas un collègue », QS 12 (1980), p. 55-81.

${ }^{62}$ CAlame, o.c. (n. 53) et "Mémoire collective et temporalités en contact : Somare et Hérodote », RHR 215 (1998), p. 350.

${ }^{63}$ En VIII, 184, Artabanos se trouve déjà justifié : pas de port pour autant de bateaux.

${ }^{64}$ Par contre, en VII, 224, 1, Hérodote ne donne pas la liste des noms des 300 Spartiates morts avec Léonidas, cette liste ne correspondant pas à l'orientation narrative. Il tient néanmoins

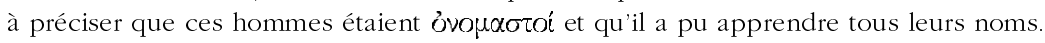


peut trouver exposée dans le dialogue entre Solon et Crésus ${ }^{65}$, où Solon, en dénombrant les jours d'une vie en un calcul arithmétique comme autant de moments de la vie humaine ouverts au changement de fortune (en décomposant par la multiplication les heures en minutes), avertit Crésus que le Temps décomposé en une infinité d'instants est plus fort que la prospérité humaine fondée sur la richesse. Tout comme Crésus qui, après avoir montré à Solon toutes ses richesses, reçoit une réponse conforme à la mesure grecque ( $\dot{\omega} c \tau \dot{\alpha}$ $\pi x \varrho ’$ ñjĩ, I, 30), Xerxès mesure la victoire par le nombre élevé de ses soldats, face à Démarate, qui y oppose à la fierté «arithmétique » du Grand Roi, l'idéal auquel les guerriers grecs se conforment et se battent jusqu'au bout (VII, 102; 104).

Fidèle à son projet, Hérodote commémore ici un thaumasion ${ }^{66}$, qui dépasse les limites humaines telles que les Grecs les conçoivent. Un tel «mouvement de populations» (pantas anthropous) ne peux qu'évoquer la Dios Boulế, dans un style tout à fait iliadique, au moment même où se déploient dans le récit les innombrables soldats de Xerxès : «Ô Zeus, pourquoi, ayant tu pris l'aspect de l'homme perse et le nom Xerxès au lieu de Zeus, veux-tu détruire la Grèce? Tu pouvais y parvenir sans tout cela ». Tournons-nous encore une fois vers Homère : «La situation de communication en catalogue se caractérise constamment dans l'Iliade par une certaine solennité liée à une conjoncture d'urgence ou de crise ». Le catalogue hérodotéen obéit à cette logique en exprimant la solennité du moment : son « expansion » narrative augure la mauvaise suite, en bâtissant la longue liste des «futurs » vaincus ${ }^{68}$. Dans l'ainos, en effet, le sens d'un passage est rendu clair par la suite des événements ${ }^{69}$.

\section{Rire d'Hécatée : Hérodote n'est pas un généalogiste}

Au début de cet article, j'ai précisé que j'envisage la généalogie comme une sous-catégorie de la forme catalogique, comme un catalogue qui déploie

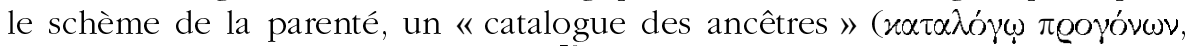
Théétète, 175a), comme le dit Platon ${ }^{70}$. Les transformations intergénériques de

\footnotetext{
${ }^{65}$ Rapprochement de l'échange entre Xerxès et Artabanos avec celui entre Solon et Crésus, WATERs, o.c. (n. 19), p. 71. Voir, plus généralement, F. HARTOG, « Myth into Logos: the Case of Croesus, or the Historian at Work », in R. BuxTon (éd), From Myth to Reason?, Oxford, 1999, p. $183-195$.

${ }^{66}$ La structure de cette unité narrative le démontre de façon paratactique, indirectement. Cette voie argumentative existe bien à côté de celles mises en évidence par C. Darbo-Peschanski et R. Thomas, et relève probablement de l'ainos (voir supra). C. HunzIGER, « La notion de $\theta \tilde{\omega} \mu \alpha$ chez Hérodote », Ktèma 20 (1995), p. 47-70.

${ }^{67}$ L. SLATKIN, The Power of Thetis, California, 1991, p. 118 sq.; P. Rousseau, « L'intrigue de Zeus », Europe 865 (mai 2001), p. 120-158.

${ }^{6}$ PAYEN, o.c. (n. 17), p. 103.

${ }^{69}$ NaGY, Pindar's Homer, cit. (n. 34), p. 248.

${ }^{70}$ À titre d'exemple, je citerai deux cas pour montrer combien il peut être artificiel de construire catalogue et généalogie en deux catégories séparées. La discussion de I. RUTHERFORD,
} 
la généalogie sont donc au cœur de notre sujet. Hérodote ne s'adonne pas à l'exercice généalogique per se, les listes généalogiques brèves qu'il expose illustrent, comme chez Homère, le rôle des personnages. Comme il tient à inscrire régulièrement dans son œuvre ce qui le différencie par rapport aux autres formes du savoir ${ }^{71}$, il exprime ses réserves à l'égard du genre généalogique dans le long exposé sur le savoir généalogique égyptien, dont fait partie le passage sur Hécatée récitant sa généalogie. Or, pour les modernes, c'est grâce au regard critique qu'Hécatée affiche au début de sa Genealogia qu'il est associé à la vague rationaliste. Cependant, le sceptiscisme à l'égard de l'information généalogique, tout comme la critique des autres traditions sont déjà perceptibles chez Homère. Une brève relecture de deux passages d'Hérodote et d'Homère, bien connus, étayera cette suggestion.

La lecture des généalogies hérodotéennes relève de la question classique si le temps est un principe structurant dans l'œuvre. Récemment Irène de Jong ${ }^{72}$ a reformulé utilement le débat sur le plan méthodologique, en qualifiant d'«anachronique » la structure chronologique de l'œuvre, preuve supplémentaire de l'influence décisive du modèle homérique. La distinction proposée par Mitchel, dans son article bien connu, entre généalogies poétiques, généalogies érudites des historiographes et chronologies arithmétiques ${ }^{73}$ aide à comprendre que, tout comme les nombreux logoi qu'il synthétise, Hérodote doit faire face à une grande variété de logiques généalogiques et chronologiques. C'est ce qu'il exprime clairement dans l'épisode d'Hécatée en Égypte. Ici il ne s'agit pas seulement de situer les événements dans le temps mais de se situer aussi par rapport aux autres logoi et œuvres.

Il faut rappeler qu'Hérodote collecte dans les temples d'Héliopolis et de Thèbes un savoir étroitement lié à la forme catalogique ${ }^{74}$. En II, 100, les

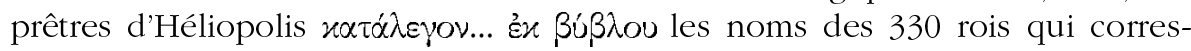
pondent à des générations humaines. Conforme à son attitude générale à l'égard de la forme catalogique, Hérodote ne s'arrêtera par la suite que sur les rois qui sont associés à des faits remarquables. À ce premier calcul du temps historique égyptien s'ajoute la contre-preuve obtenue par les archiereis du temple de Zeus Ammon à Thèbes, qui introduisent Hérodote - comme il l'avaient fait avec Hécatée - dans le megaron où sont dressés les colosses

l.c. (n. 15) autour de la poésie catalogique (notamment les Ehoiai) comme genre montre la flexibilité de passage d'une liste des noms agencés selon un critère précis (les femmes qui ont couché avec un dieu) à un catalogue de nature généalogique. Le Catalogue des Vaisseaux homérique comporte également une composante généalogique.

${ }^{71}$ Voir FOwLER, l.c. (n. 20) : le traitement narratif des sources constitue la plus grande originalité d'Hérodote; aussi MARINCOLA, l.c. (n. 18).

72 DE JONG, l.c. (n. 33), p. 252 sq. Voir aussi F. MiTCHEL, « Herodotus' Use of Genealogical Chronology », Phoenix 10 (1956), p. 48 et Darbo-Peschanski, o.c. (n. 19), p. 25 sq

${ }^{73}$ Ce qui finalement reflète les divergences narratives de l'historiographie naissante : orientation chronologique/ généalogique ou poétique/biographique.

${ }^{74}$ Voir le commentaire pertinent de CALAMe, l.c. (n. 62), p. 355 sq. Voir aussi Mitchell, l.c. (n. 72); J. COBET, «The Organization of Time in the Histories », in BAKKER et al., o.c. (n. 16), p. $387 s q$ 
représentant les grands prêtres qui les ont précédés et qui y ont érigé leur statue de leur vivant, traçant dans l'espace un «catalogue » des générations égyptiennes en «images», en trois dimensions. Tout en énumérant les statues, les grands prêtres ont récité leur généalogie qui remontait à 345

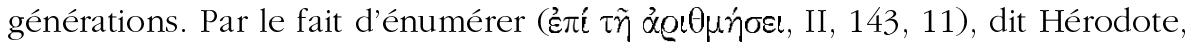
ils ont produit une généalogie qui contredisait (antigeneêlogein) celle d'Hécatée. Aussi longtemps que l'énumération des prêtres pouvait remonter, jusqu'à la $345^{\mathrm{e}}$ génération $^{75}$, il ne paraissait pas possible de rattacher une généalogie humaine à un dieu ou un héros.

Cette nouvelle prestation catalogique, qui répond à la généalogie grecque récitée par Hécatée, est une confrontation de deux pratiques savantes, dont une possède une écrasante supériorité fondée sur l'ancienneté et sur la méthode suivie, visant à prouver, par l'ancienneté imposante de la documentation égyptienne, le vice caché de la généalogie présentée par Hécatée, aboutissant à une mise en cause globale du système généalogique grec. Les

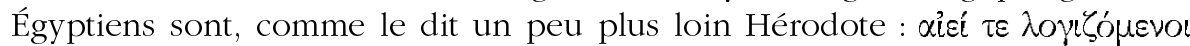

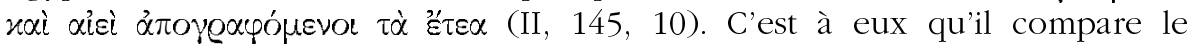
système généalogique grec pour relativiser sa validité, choisissant des porteparoles identifiables et prestigieux. Cette critique implicite illustre bien les distinctions pertinentes de Mitchel, puisque nous avons ici une confrontation entre généalogie mythique et généalogie chronologique (etea / geneai traduisent cette opposition dans le texte même ${ }^{76}$ ). Le système égyptien représente fidèlement le sens énumératif du katalegein, tandis que la généalogie mythique se fonde sur la hiérarchisation temporelle de proteron, busteron.

Dans ce contexte, Hécatée devient une cible paradigmatique, à cause de sa notoriété assurément mais aussi en tant qu'exemple personnifié d'une pratique généalogique grecque peu sérieuse ${ }^{77}$ : la critique vise donc toute une forme de savoir ${ }^{78}$. En effet, si l'on tient compte du style paratactique de ses procédés demonstratifs, Hérodote rattache l'épisode à une critique plus fondamentale touchant à l'ensemble du système généalogique grec relatif aux dieux, concluant sur l'hypothèse bien connue : que les Grecs auraient appris tardivement les noms de certaines divinités (Dionysos, Héraclès, Pan), et ce n'est qu'à partir de ce moment-là qu'ils auraient commencé la généalogie

\footnotetext{
75 Drews, o.c. (n. 18), p. 56 sq.; A. Lloyd, Herodotus, Book II : Commentary 99-182, Leiden, 1988

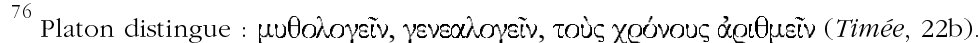

77 Je ne peux pas être d'accord avec D. BOEDEKER, «Herodotus' Genre(s)», in DEPEW OBBink, o.c. (n. 15), p. 108 : «These three references to Hecateaus as a compiler of historical and geographical information are directed to specific rather than generic concerns. »

${ }^{78} C f$. IV, 36, 2. Sur ce passage et sur l'esprit agonistique de l'usage de la première personne par Hérodote, voir BOEKFKer, l.c. (n. 77), p. 106-107 et supra. Hécatée « cataloguant ", essaie de convaincre les alliés d'Aristagoras de ne pas se rebeller contre les Perses, en énumérant les

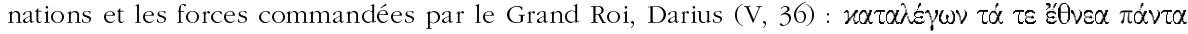

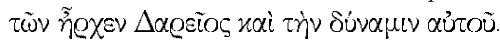


relative à eux (II, 146, 2) $)^{79}$. L'épisode autour d'Hécatée contribue à mettre en lumière la faiblesse de cette mémoire courte et du genre discursif qui en perpétue les erreurs.

\section{Homère non plus n'est pas généalogiste}

Le peu d'interêt qu'Homère montre pour les filiations a déjà été remarqué. Subordonnée au principe de la mimésis, dépourvue donc de l'écran d'objectivité qu'offre le discours indirect, la généalogie est prononcée par les personnages et obéit à l'esprit polémique caractéristique de l'épopée. En effet, les mises en scène des généalogies laissent apparaître une attitude cohérente, tout à fait réfléchie, de la part du poète. Dans l'Iliade, elles sont, tout d'abord, toujours integrées dans les discours; ensuite, elles sont presque toujours courtes et remontent au plus à l'arrière grand-père; enfin, les ancêtres hors du temps épique ne sont pas mentionnés; en matière de généalogie, les êtres merveilleux, des temps antérieurs à l'âge héroïque, n’intéressent pas l'épopée $^{80}$.

Cependant, il n'a pas fallu attendre Platon qui se moque des longs « kata$\log o i$ » d'ancêtres de toutes origines, pour avoir une critique à l'égard de l'usage rhétorique de la généalogie : « ... aïeux et bisaieux, chacun les a par myriades, myriades qu'on ne saurait nombrer, où riches et gueux, rois et esclaves, Barbares et Hellènes, ont eu dix mille et dix mille fois leur tour en la lignée de n'importe qui » (Théétète, 175a). Quand Athéna-Mentès fait semblant de s'enquérir de l'identité de Télémaque, c'est ainsi que le jeune homme déboussolé lui répond: «qui peut connaître sa lignée? Ma mère dit que je suis le fils d'Ulysse ${ }^{81}{ }^{81}$, Chez Homère, le concerné qui énonce lui-même sa généalogie l'accompagne souvent d'expressions de réserve ou en fait un objet de polémique ${ }^{82}$.

Le discours généalogique d'Énée (XX, 203-205; 244 sq.) illustrera bien cette attitude critique, puisque sa longueur « exceptionnelle » appelle un commentaire sur la poétique même ${ }^{83}$. La généalogie des princes troyens aurait pu être livrée sur le ton objectif de la diégèse. Il n'en est rien. Son énonciation résulte d'une intervention apollinienne qui met Énée face au meilleur des Achéens, Achille, sorti sur le champ de bataille chercher Hector pour venger Patrocle.

\footnotetext{
${ }^{79}$ R. OsBORNE, «Archaic Greek History », in BAKKER et al., o.c. (n. 16), p. 500.

J.-C. CARRIÈRE, « Du mythe à l'histoire. Généalogies héroïques, chronologies légendaires et historicisation des mythes », in D. AUGER, S. SAÏD (éds), Généalogies mythiques, Paris, 1998, p. 5565. A. Ballabriga, «Hérodote et l'histoire de l'épopée », in C. Darbo-Peschanski (éd.), Constructions du temps dans le monde grec ancien, Paris, 2000, p. 325-339.

${ }^{81}$ I, 215-216. Voir les remarques importantes de A. FORD, Homer. The Poetry of the Past, Ithaca / London, 1992, et I. PAPADOPOUlou-BelmeHDI, « Maternités homériques : la 'mauvaise mère' de Télémaque », Itaca 16-17 (2001), p. 43-59.

${ }^{82}$ Iliade $\mathrm{V}, 635$ : le fils d'Héraklès Tlépolème met en question l'ascendance de Sarpédon, fils de Zeus.

${ }^{83}$ Gr. NAGY, The Best of the Achaeans, Baltimore / London, 1979, p. 265-275, montre que le vocabulaire encadrant cette généalogie se réfère à des traditions poétiques.
} 
D'emblée, du point de vue iliadique, l'usage - rusé - des arguments généalogiques par Apollon sonne ironiquement: "Tu es né d'Aphrodite, fille de Zeus... lui est né d'une déesse inférieure (105-106)». Malgré ses objections premières devant l'invincibilité d'Achille, Énée se laisse entraîner. Face à face avec le meilleur des Achéens, il récite la plus longue généalogie de l'Iliade ${ }^{84}$. Le poète signale l'«irrégularité » de cette longeur au sein du projet épique par un nombre important de vers qui commentent le langage généalogique : «... on ne nous verra pas revenir du combat ayant réglé notre querelle tout bonnement avec des mots enfantins. Si pourtant tu veux apprendre davantage et savoir ma naissance - nombreux sont déjà ceux qui la connaissent écoute... »

Suit la récitation généalogique propre, qu'Énée conclut ainsi : «Allons, ne restons pas là à parler comme des enfants, alors que nous sommes en pleine mêlée et carnage... Sommes-nous forcés de nous disputer, de nous prendre à partie ainsi face à face, comme des femmes en colère que l'esprit de querelle qui dévore les cours fait aller en pleine rue se prendre à partie et se lancer mutuellement autant de mensonges que de vérités, le dépit leur dictant les uns comme les autres? Ce n'est pas avec des mots que tu détourneras de toi mon courage impatient... » Dans le vaste champ des traditions orales (eُ $\pi \dot{\varepsilon} \omega \nu$

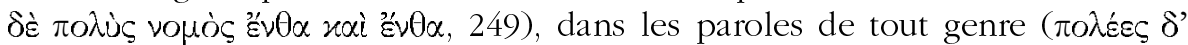

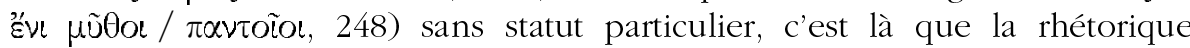
homérique inscrit le discours généalogique d'énée, tout comme l'exhortation généalogique que lui avait adressé Apollon et qui sera tristement démentie par l'issue du duel.

Prêtées à Énée, les paroles du poète expriment plutôt un procédé épique récurrent : énoncer indirectement la délimitation thématique de l'Iliade et son

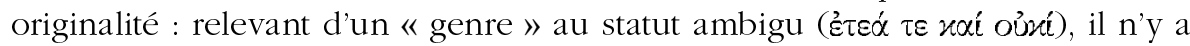
pas de discours généalogique important chez Homère qui ne soit pas accompagné d'expressions de réserve : "Pourquoi veux-tu connaitre ma lignée ?», dit Glaukos à Diomède et suit la magnifique image des générations humaines qui sont semblables aux feuilles des arbres et qui apparaissent et périssent selon le cycle de la nature. Par contre, l'axe hiérarchique qui organise la matière et l'éthique épique est clairement indiqué par Énée : «Voici la race, le sang dont je me flatte d'être issu. Mais, s'il s'agit de courage, c'est Zeus seul qui, chez les hommes, le fait, à son gré, grand ou petit, parce qu'il est toutpuissant. » Transformée en discours agonistique ${ }^{85}$, consubstantiel au comportement héroïque, la généalogie dans l'Iliade se tisse au cour de l'action. L'épopée trie fortement dans le savoir généalogique traditionnel et adapte les « citations » génériques. Le thème digne d'être commémoré et qui la constitue en genre ${ }^{86}$, ce sont bien les klea andrôn, le présent épique des générations

\footnotetext{
${ }^{84}$ P. WATHelet, « La généalogie de Priam », in AUGER - SAÏD, o.c. (n. 80), p. 177 sq., et P. BRillet-Dubois, «Généalogie et géographie troyennes dans le discours d'Énée au chant XX de l'Iliade », ibid., p. 189 sq.

${ }^{85}$ Voir les remarques importantes de MARTin, o.c. (n. 40), p. 126-130.

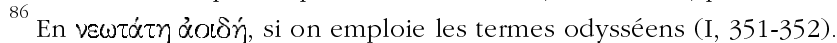


héroïques $^{87}$. Ne pas raconter le monde dans l'ordre catalogique d'une généalogie, privilégier les erga glorieux de cette génération épique, telle est la structure anthropocentrique d'un récit non linéaire qu'Homère a légué à Hérodote.

Ces transformations profondes du mode catalogique annoncent une nouvelle conception de ce que peut être l'unité narrative et le rapport entre forme et thème. Entre les mains du « divin » Homère, la progression linéaire et le souci d'exhaustivité sont repensés et c'est là une des raisons majeures de l'admiration qu'exprime Aristote $^{88}$ : au sein d'un projet strictement sélectif,

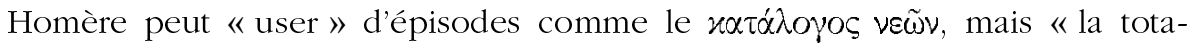
lité » ne le tente pas. Le défi à la linéarité ou à la successivité est inscrit au prooimion même de l'Odyssée : «choisis ton point de départ, Muse, pour nous raconter l'homme errant... " C'est cette conception nouvelle de l'unité narrative, de la successivité et du rapport entre forme et contenu, qui sera tout à fait décisive dans le processus de la formation de différents genres discursifs en Grèce ancienne ${ }^{90}$.

Université libre de Bruxelles

Ioanna PAPADOPOULOU

Centre de Philosophie ancienne / CP 175

50, avenue F.D. Roosevelt

B - 1050 BRUXELLES

Courriel : Ioanna.Papadopoulou@ulb.ac.be

\footnotetext{
${ }^{87}$ CARRIÈre, l.c. (n. 80), p. 68 : « Pour le poète de l'Iliade, ce qui est digne de mémoire, les klea andrôn, ce sont les exploits guerriers des dernières générations héroïques. »

${ }^{88}$ HARTOG, o.c. (n. 5), p. 50 : « La généalogie est une écriture qui, une fois lancée, peut se développer indéfiniment. Il manquera toujours des noms... » L'espace manque pour commenter ici l'attitude d'Aristote à l'égard d'Hérodote et plus généralemet du genre historiographique; voir, entre autres, M. FINLEY, "Mythe, mémoire et histoire », in Mythe, mémoire, histoire, Paris, 1981 (trad. fr.), p. 9 sq. Sur l'historien et le poète entre Aristote et Ricœur, voir S. KLIMIs, Le statut du mythe dans la Poétique d'Aristote, Bruxelles, 1997, p. 25 sq.; Cl. Calame, "Pour une anthropologie des pratiques historiographiques ", L'Homme 173 (2005), p. 11-46; PAYEN, l.c. (n. 17).

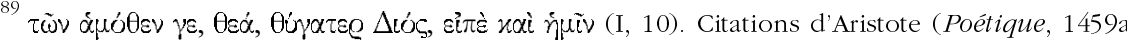

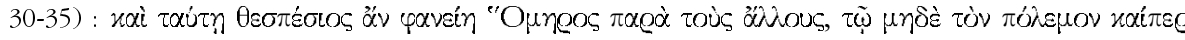

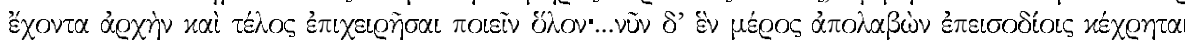

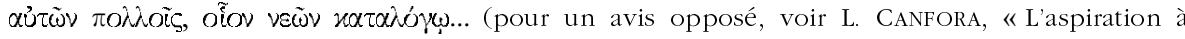
l'exhaustivité dans l'épopée et l'historiographie, Europe 865 [mai 2001], p. 102-119). Cf. aussi W. MinTON, «Invocation and Catalogue in Hesiod and Homer », TAPhA 93 (1962), p. 204.

${ }^{90}$ Je remercie vivement Claude Calame, Lambros Couloubaritsis et Pascal Payen de leurs précieux commentaires.
} 